\title{
Cirurgia monocular para esotropias de grande ângulo: histórico e novos paradigmas
}

\author{
Monocular surgery for large-angle esotropias: review and new paradigms
}

Edmilson Gigante ${ }^{1}$, Harley Edson do Amaral Bicas ${ }^{2}$

\section{RESUMO}

As primitivas cirurgias de estrabismo, as miotomias e as tenotomias, eram feitas, simplesmente, seccionando-se o músculo ou o seu tendão, sem nenhuma sutura. Estas cirurgias eram feitas, geralmente, em um só olho, tanto em pequenos como em grandes desvios e os resultados eram pouco previsíveis. Jameson, em 1922, propôs uma nova técnica cirúrgica, usando suturas e fixando, na esclera, o músculo seccionado, tornando a cirurgia mais previsível. Para as esotropias, praticou recuos de, no máximo, $5 \mathrm{~mm}$ para o reto medial, o que se tornou uma regra para os demais cirurgiões que o sucederam, sendo impossível, a partir daí, a correção de esotropias de grande ângulo com cirurgia monocular. Rodriguez-Vásquez, em 1974, superou o parâmetro de 5 mm propondo amplos recuos dos retos mediais ( 6 a $9 \mathrm{~mm}$ ) para o tratamento da síndrome de Ciancia, com bons resultados. Os autores revisaram a literatura, ano a ano, objetivando comparar os vários trabalhos e, com isso, concluíram que a cirurgia monocular de recuo-ressecção pode constituir uma opção viável para o tratamento cirúrgico das esotropias de grande ângulo.

Descritores: Estrabismo/história; Estrabismo/cirurgia; Esotropia/cirurgia Músculos oculomotores/cirurgia; Acuidade visual; Procedimentos cirúrgicos oftalmológicos

\begin{abstract}
The primitive strabismus surgeries, myotomies and tenotomies, were performed simply by sectioning the muscle or its tendon without any suture. Such surgeries were usually performed in just one eye both in small and in large angles with not really predictable results. In 1922, Jameson introduced a new surgery technique using sutures and fixing the sectioned muscle to the sclera, increasing surgery predictability. For the esotropias he carried out no more than $5 \mathrm{~mm}$ recession of the medial rectus, which became a rule for the surgeons who followed him, which made it impossible from then on to correct largeangle esotropias with a monocular surgery. Rodriguez-Vásquez, in 1974, exceeded the $5 \mathrm{~mm}$ parameter by proposing large recessions of the medial recti (6 to $9 \mathrm{~mm}$ ) to treat the Ciancia syndrome with good results. The authors revised the literature year after year with the purpose of comparing the several works and concluded that monocular recession-resection surgery may be a feasible option for the surgical treatment of large-angle esotropias.
\end{abstract}

Keywords: Strabismus/history; Strabismus/surgery; Esotropia/surgery; Oculomotor muscles/surgery; Visual acuity; Ophthalmologic surgical procedures

\section{INTRODUÇÃO}

$\mathrm{H}$ istoricamente, na moderna estrabologia, as esotropias de grande ângulo têm sido corrigidas cirurgicamente atuando-se nos quatro músculos horizontais ou, pelo menos, em três deles (ambos os olhos), visto que a grande maioria dos autores ainda considera um dogma, da estrabologia mundial, fazerem-se recuos para os retos mediais de, no máximo, $5 \mathrm{~mm}$, alegando-se que recuos maiores dariam grandes limitações dos movimentos oculares e grande comprometimento da convergência ${ }^{(1-5)}$. Realmente, com recuos tão pequenos, não seria possível, nas cirurgias binoculares, uma correção cirúrgica satisfatória, para esses casos, atuando-se somente em dois músculos. $O$ máximo que se poderia corrigir, com recuos de $5 \mathrm{~mm}$ de ambos os retos mediais, seria $30^{4}$, em média(6). Daí a

\footnotetext{
Trabalho realizado na Faculdade de Medicina de Presidente Prudente, Universidade do Oeste Paulista - UNOESTE - Presidente Prudente (SP), Brasil.

Médico, Departamento de Oftalmologia, Faculdade de Medicina de Presidente Prudente, Universidade do Oeste Paulista - UNOESTE - Presidente Prudente (SP), Brasil.

Médico, Departamento de Oftalmologia, Otorrinolaringologia e Cirurgia de Cabeça e Pescoço, Faculdade de Medicina de Ribeirão Preto, Universidade de São Paulo - USP - Ribeirão Preto (SP), Brasil.

Endereço para correspondência: Edmilson Gigante. Av. Washington Luiz, $874-1^{\circ}$ Andar - Presidente Prudente (SP) - CEP 19015-150 - E-mail: egigante@stetnet.com.br

Recebido para publicação em 28.08.2007

Última versão recebida em 08.02.2010

Aprovação em 08.03.2010

Este atualização corresponde à parte inicial (introdução e revisão bibliográfica) da dissertação de mestrado do autor, que foi defendida em 09/05/2008, no Departamento de Oftalmologia, Otorrrinolaringologia e Cirurgia de Cabeça e Pescoço, Faculdade de Medicina, Ribeirão Preto, Universidade de São Paulo, tendo como orientador o Prof. Dr. Harley Edson do Amaral Bicas.
}

necessidade de atuar-se, também, nos retos laterais, fazendose ressecções nos dois músculos ou, pelo menos, em um deles.

Por outro lado, com relação às ressecções dos retos laterais, os oftalmologistas têm sido um pouco mais generosos, a maioria aceitando valores máximos um pouco maiores, geralmente em torno de $10 \mathrm{~mm}^{(2,45,7)}$. Dessa forma, usando-se a cirurgia monocular de recuo-ressecção para o tratamento das esotropias de grande ângulo, seria possível uma correção um pouco maior do que aquela conseguida com o duplo recuo dos retos mediais, mas, mesmo assim, não se conseguiria corrigir mais do que 45 a $50^{\Delta}$, com um recuo máximo de $5 \mathrm{~mm}$ do reto medial e uma ressecção máxima de $10 \mathrm{~mm}$ do reto lateral ${ }^{(6)}$.

Portanto, com relação ao tratamento cirúrgico dos esodesvios, pode-se inferir que, tanto na cirurgia binocular como na monocular, não se conseguem correções maiores, atuando-se somente em dois músculos, por causa do limite máximo, imposto, de $5 \mathrm{~mm}$ no recuo do reto medial. Ultrapassando-se esse limite, provavelmente poder-se-ia conseguir correções maiores, possivelmente acima das $50^{\Delta}$. O presente trabalho pretende, inicialmente, mediante ampla revisão bibliográfica, fazer uma pesquisa a respeito do estabelecimento do parâmetro de $5 \mathrm{~mm}$, como recuo máximo do reto medial, procurando entender suas causas e suas consequências na cirurgia estrabológica, bem como pesquisar, ainda, a existência de trabalhos científicos, na literatura mundial, nos quais tenham sido praticados recuos do reto medial maiores que $5 \mathrm{~mm}$.

\section{HISTÓRICO}

Até o século XVIII as tentativas de tratamento de pacientes estrábicos ficaram no campo do que se poderia chamar de 
tratamento clínico, com resultados, esperadamente, muito pouco expressivos ${ }^{(8)}$.

Foi em meados daquele século que, pela primeira vez, surgiu um tratamento que, embora muito rudimentar, pode ser chamado de tratamento cirúrgico. Refere-se aqui às cirurgias realizadas, em série, por um personagem muito polêmico e muito conhecido, na época, aclamado por muitos, mas também odiado por outros tantos. Trata-se de John Taylor ${ }^{(8-11)}$ que fazia uma cirurgia, conhecida, na época, como "estrabotomia" ou "neurotomia"(8). Não há consenso histórico sobre sua técnica, mas é bem provável que ele seccionasse o músculo mais forte, causador do estrabismo. Teve, na época, muitos seguidores, mas os maus resultados foram colocando esta técnica no esquecimento.

Após a época de Taylor, os relatos históricos sobre cirurgia de estrabismo, somente voltam a aparecer no século seguinte, quando Dieffenbach opera, em 25 de outubro de 1839, em Berlim, um menino de sete anos de idade, seccionando seu reto medial e corrigindo, com sucesso, seu estrabismo convergente ${ }^{(8-9,11-12)}$. Esta técnica ficou conhecida como miotomia e difundiu-se por toda a Europa e também para as Américas, incluindo o Brasil, que teve um ilustre representante, com tese de doutorado defendida com esta técnica cirúrgica ${ }^{(13)}$. O otimismo em relação à miotomia foi causado pela grande melhora estética dos pacientes a curto prazo, pois, uma vez seccionado, totalmente, o músculo hiperfuncionante, o olho estrábico deslocavase para uma posição, relativamente, central, "curando" o estrabismo. Entretanto, com o passar do tempo foram aparecendo as complicações, em parte devidas ao material grosseiro utilizado nas cirurgias e à falta de assepsia adequada, mas, principalmente, porque a grande maioria dos casos passou a apresentar um estrabismo divergente secundário, esteticamente pior que o anterior, o que acabou por inviabilizar esse tipo de cirurgia.

Após os trabalhos de Bonet, em 1841, sobre a anatomia dos músculos oculares externos, foi possível dissecar melhor os músculos, localizando-se suas inserções, surgindo assim uma nova técnica cirúrgica, a tenotomia, na qual seccionava-se o tendão e não o corpo do músculo como se fazia na miotomia. Destacaram-se, aqui, os trabalhos de *Graeffe $1857^{(14)}$, que foram fundamentais para consolidar, definitivamente, a nova cirurgia $^{(9,11-12,15)}$. Apesar de apresentar, inicialmente, bons resultados, com o passar do tempo os estrabismos divergentes secundários voltaram a aparecer, pois o tendão seccionado, geralmente, não se fixava na esclera, ficando, o músculo, totalmente sem força, à mercê da contração do reto lateral. Mesmo nos casos em que o tendão se fixava na esclera, nunca se sabia onde ele iria "parar", tornando a cirurgia imprevisível ${ }^{(9,12)}$. Como tentativa de salvar esta técnica, tentou-se, ainda, a tenotomia parcial mas os resultados não foram satisfatórios $(9,12,16)$.

Em face dos maus resultados com a tenotomia (tanto total como parcial) os oftalmologistas da época voltaram-se para uma nova cirurgia, realizada pela primeira vez, em 1855, por Chritchet, na Inglaterra, o avançamento muscular. Tratava-se de uma cirurgia, ao contrário de suas antecessoras, que tinha a finalidade de aumentar a força do músculo operado, avançando a inserção do reto lateral e suturando-o próximo ao limbo. Pela primeira vez, na cirurgia de estrabismo, foram utilizadas as suturas, pois, antes, nas tenotomias e nas miotomias, simplesmente seccionava-se o músculo ou o seu tendão(9,12).

Em pouco tempo, essa cirurgia ganhou a preferência dos cirurgiões, mas ela também tinha os seus problemas, pois o simples avançamento de um reto lateral corrigia somente pequenos desvios. Passou-se, então, a ressecar uma parte do músculo,

* Graefe AV. Archiv F Ophtalmologie. 3:342-4. 1857 apud $^{(14)}$ antes de avançá-lo, para obter-se uma maior correção(12). Estava criada a cirurgia de ressecção muscular, tão utilizada, até hoje, nas correções cirúrgicas do estrabismo. Ainda assim, nos grandes ângulos, a correção era insatisfatória e, pela primeira vez na história da estrabologia, passou-se a preconizar a cirurgia bilateral, no caso, de avançamento (com ou sem ressecção) de ambos os retos laterais ${ }^{(12,17)}$.

Ainda, assim, os resultados eram discretos nos grandes ângulos, pois o reto medial não operado, continuava forte $e$ hiperfuncionante, malogrando os resultados cirúrgicos. Foi quando alguns autores passaram a preconizar (para os grandes ângulos) a cirúrgica simultânea, no mesmo olho, de uma tenotomia do reto medial associada a um avançamento do reto lateral, com ou sem ressecção ${ }^{(12,14,18)}$. Esta técnica apresentava a vantagem de enfraquecer o músculo hiperfuncionante (no caso, o reto medial) e fortalecer o hipofuncionante (o reto lateral), além de se operar somente um olho, certamente o olho estrábico e, provavelmente, amblíope, pois naqueles tempos o tratamento sensorial ainda era muito rudimentar. Estava iniciando-se uma polêmica que, até os nossos dias, ainda não foi resolvida, qual seja a opção pela cirurgia monocular ou binocular, principalmente nos grandes desvios. Acrescente-se, ainda, que alguns autores, certamente a minoria, utilizavam a cirurgia binocular, fazendo-se a tenotomia em ambos os retos mediais ${ }^{(12)}$.

\section{NOVOS PARADIGMAS}

No final do século XIX, a cirurgia de estrabismo entrava, novamente, em um impasse: embora fosse a preferida dos oftalmologistas, a cirurgia de avançamento muscular (com ou sem ressecção) mostrava-se insatisfatória para a correção dos grandes desvios convergentes, mesmo quando realizada em ambos os olhos. Desse modo, acabava, geralmente, sendo necessário combinar-se, no mesmo olho, o avançamento com a tenotomia, sendo que esta última já estava, nesta época, caindo em descrédito devido ao grande número de desvios divergentes secundários, agravados, ainda mais, na associação com o avançamento, devido ao fortalecimento do reto lateral| ${ }^{(12,14)}$. Em resumo, o problema estava no fato de que a tenotomia era uma cirurgia muito imprevisível, porque nunca se sabia, exatamente, onde o músculo seccionado iria fixar-se na esclera e, consequentemente, nunca se sabia com certeza, quanto do desvio inicial seria corrigido ${ }^{(16)}$. A solução desse impasse somente poderia advir de uma nova técnica, que possibilitasse a fixação do músculo na esclera, a uma distância previamente calculada, em relação à inserção original.

\section{RETROINSERÇÃO COM FIXAÇÃo ESCLERAL}

Alguns autores tentaram solucionar esse problema, entre eles Prince, Jackson e Curdy, mas foi somente com Jameson, em $1922^{(19)}$ que esses problemas foram finalmente resolvidos, pois esse autor desenvolveu uma nova técnica, que consistia em suturar o músculo seccionado, na esclera, a uma certa distância de sua inserção original, impedindo, assim, seu deslizamento, tornando, desse modo, a cirurgia bastante previsível(10,20-23). Com esta nova técnica, o autor relata que teve duas preocupações: o cuidado em não perfurar a esclera com a sutura e qual o montante do recuo do músculo operado a ser feito, a partir de sua inserção original. Com relação à primeira, usou agulhas especiais (futuramente chamadas de "agulhas de Jameson") e recomendou fazer pontos sem transfixar a esclera. Com relação à segunda, tomou o equador do globo ocular como limite máximo para o retrocesso do músculo seccionado. Como a distância entre a inserção do reto medial e o equador é, em média, de 5 a $6 \mathrm{~mm}$, o autor passou a recomendar um recuo máximo de $5 \mathrm{~mm}$, para o reto medial, em suas cirurgias ${ }^{(19)}$. Achava ele 
que, com recuos maiores, além do equador, o músculo perderia totalmente sua força, funcionaria como um músculo paralítico e, consequentemente, os desvios divergentes secundários novamente aconteceriam (como na miotomia e na tenotomia).

\section{UM PARADIGMA TEÓRICO}

A partir dessa referência de Jameson, todos os cirurgiões que passaram a usar a nova técnica, obedeceram a essa regra, tomando como verdadeiro dogma, da cirurgia do reto medial o recuo máximo de $5 \mathrm{~mm}$, regra essa que, até hoje, raramente é contrariada. Alguns autores até exageraram nesta precaução, indicando, em suas cirurgias, valores máximos ainda menores do que $5 \mathrm{~mm}^{(5,16,24)}$. Com relação a Prince, Jackson e Curdy, também citados, na literatura, como possíveis pioneiros da cirurgia de recuo muscular com sutura escleral, não foram encontrados relatos mais minuciosos de suas técnicas, principalmente no sentido de se saber quantos milímetros, de recuo do reto medial, praticaram em suas cirurgias. Entretanto, pelo que foi descrito anteriormente, pode-se inferir que, provavelmente, não tenham feito recuos maiores do que $5 \mathrm{~mm}$, em seus primeiros pacientes. Seria muita ousadia para aquela época.

Como se percebe dos relatos anteriores, o paradigma do recuo máximo de $5 \mathrm{~mm}$ para o reto medial, não foi estabelecido na prática, após serem feitas cirurgias com recuos de 6, 7, 8 ou mais milímetros, para chegar-se a um valor ideal (e possível), após análise dos resultados encontrados. Foi, na realidade, um parâmetro estabelecido teoricamente, com base no medo de que, ultrapassando o equador do olho, aparecessem os desvios divergentes secundários, tão temidos naqueles tempos. Mas, a ciência não pode sustentar-se em cima de pilares teóricos; ao contrário, deve basear-se em trabalhos científicos que mostrem, na prática, a verdade, abandonando os dogmas e as pseudoverdades, pois sabe-se, muito bem, que os cientistas trabalham com verdades relativas, transitórias e sempre passíveis de serem modificadas.

\section{UM PARADIGMA PRÁTICO}

O mexicano Rodolfo Rodriguez Vasquez surpreendeu os estrabólogos presentes ao IV Congresso do Conselho Latinoamericano de Estrabismo (CLADE), realizado no México de 12 a 16 de maio de 1974, quando apresentou uma idéia revolucionária para o tratamento cirúrgico da esotropia congênita com limitação bilateral de abdução, também denominada síndrome de Ciancia, através de amplos recuos dos retos mediais de ambos os olhos. Até aquela data, esta síndrome era tratada operando-se os quatro músculos retos horizontais ou pelo menos três deles, visto que o ângulo de desvio desse tipo de esotropia, dificilmente é menor do que $50^{\Delta}$, ultrapassando, na maioria das vezes, as $60^{\Delta}$. Assim sendo, se fossem feitos recuos de retos mediais de, no máximo, $5 \mathrm{~mm}$, operando-se somente os retos mediais de ambos os olhos, certamente ter-se-ia, como resultado, sempre uma hipocorreção. Daí a necessidade de operar-se sempre três ou quatro músculos fazendo-se, também, a ressecção de um ou de ambos retos laterais ${ }^{(25)}$.

Em seu trabalho, denominado "Retroinserción amplia en cirugia de endotropias alternas", Rodríguez-Vasquez apresentou 26 casos operados da seguinte maneira:

1. Em dois casos (entre 40 e 50 ${ }^{\Delta}$ ) foram feitos recuos de $6 \mathrm{~mm}$ nos retos mediais de ambos os olhos;

2. Em quatro casos (entre 50 e $60^{\Delta}$ ) os recuos dos retos mediais foram de $7 \mathrm{~mm}$

3. Em dezoito casos (entre 65 e $75^{\Delta}$ ), recuos de $8 \mathrm{~mm}$ em ambos retos mediais;

4. Em dois casos (maiores do que $75^{\Delta}$ ) recuos de $9 \mathrm{~mm}$ nos dois retos mediais.
O autor teve 21 casos (80,27\%) com ortotropia cirúrgica (ângulo de desvio igual ou menor que $\left.10^{\Delta}\right)$, quatro casos $(15,4 \%)$ com endotropia residual e um caso $(3,8 \%)$ com exotropia residual. Em nenhum dos pacientes operados observou-se limitação da função dos retos mediais. O ponto próximo de convergência (PPC), foi normal em todos os pacientes, exceto em um, de 7 anos de idade, no qual foi feito recuo bilateral de $7 \mathrm{~mm}$, que apresentou PPC de $9 \mathrm{~cm}$.

Como se vê, a tese de que, ao ultrapassar o equador do olho nas cirurgias de recuo, o reto medial perderia sua função, não se concretizou. Ao contrário, mostrou-se que, apesar dos amplos recuos praticados, o reto medial continuava bem funcionante. Tinha-se, agora, um novo paradigma nas cirurgias de esotropia de grande ângulo e com a vantagem de ser um paradigma extraído da prática, da experiência e não um parâmetro teórico, como o antigo. Estava dado o primeiro passo para uma grande revolução na cirurgia dos esodesvios, principalmente nos grandes ângulos.

Nos anos seguintes, muitos autores testaram a idéia de Rodriquez-Vasquez pelo mundo todo ${ }^{(26-32)}$ e em todos os casos a conclusão foi sempre a mesma: os retos mediais, mesmo inseridos além do equador do olho, continuavam com sua função de convergência normal e não eram significativas as limitações laterais dos olhos operados. Entretanto, apesar dos vários trabalhos mostrando a eficácia dos grandes recuos dos retos mediais, ainda hoje alguns autores, principalmente norte-americanos, não aceitam essas cirurgias e não as utilizam, preconizando, para o tratamento cirúrgico da síndrome de Ciancia, a atuação nos quatro músculos retos horizontais numa mesma cirurgia ou, pelo menos, em três deles ${ }^{(23,33)}$. Isso mostra claramente o lado conservador da ciência, que resiste às mudanças de paradigmas, mesmo após serem mostrados trabalhos científicos demonstrando a queda dos velhos padrões.

\section{CIRURGIA MONOCULAR VERSUS CIRURGIA BINOCULAR}

Historicamente, a cirurgia de estrabismo, nos seus primórdios, foi sempre monocular, porque a miotomia e a tenotomia (sem falar na "estrabotomia") eram cirurgias que se bastavam, para as soluções dos problemas daquela época e também porque não existiam outras técnicas cirúrgicas. Basicamente nos estrabismos convergentes seccionava-se o reto medial do olho estrábico e nos estrabismos divergentes seccionava-se o reto lateral.

Na época da miotomia fazia-se a secção simples do músculo, para os desvios menores e a secção, seguida de grandes debridações, para os desvios maiores, de maneira que, operando-se somente um olho (e somente um músculo), tentavase resolver todos os casos cirúrgicos de estrabismo. Também não se deve esquecer, que nessa época ainda não existia anestesia e, por isso, era plenamente justificável que se tentasse resolver os problemas operando-se somente um olho, pois, atuando-se em ambos os olhos, o sofrimento do paciente seria dobrado $(12,14)$

Com a passagem da miotomia para a tenotomia, pouca coisa modificou-se e a conduta continuou sendo a mesma, ou seja, tenotomia simples para os desvios menores e tenotomia com grandes debridações, para os desvios maiores, mantendo-se, ainda, a cirurgia monocular para a grande maioria dos casos.

Com o aparecimento de uma nova técnica cirúrgica, a do avançamento muscular, descoberta por Critchet, em 1855, as coisas começaram a tomar um novo rumo. A nova técnica foi bem recebida pelos cirurgiões, pois as complicações da tenotomia já eram bem evidentes (quase as mesmas da miotomia) e o avançamento muscular passou a representar uma opção 
nova, uma nova esperança no tratamento cirúrgico do estrabismo. A técnica era simples de ser executada e espalhou-se, rapidamente, no meio estrabológico, passando a ser, em pouco tempo, praticada pela grande maioria dos cirurgiões. Entretanto, logo observou-se que o avançamento de um músculo corrigia somente os desvios pequenos, por maiores que fossem esses avançamentos. Mesmo posteriormente, quando se acrescentou a ressecção de parte do músculo, na cirurgia do avançamento, o problema ainda persistiu. Foi quando, pela primeira vez, os cirurgiões sentiram que a cirurgia binocular era, realmente, uma necessidade e não somente uma escolha pessoal de cada cirurgião. Passou-se então, nos grandes desvios convergentes, à prática do duplo avançamento (com ou sem ressecção) do reto externo de ambos os olhos ${ }^{(12)}$.

Porém, também essa conduta mostrou, em breve, não ser uma panacéia, pois, nos grandes desvios convergentes, o reto medial não operado permanecia muito forte e impedia que o olho chegasse à posição central somente com o reforço dos retos laterais, o que tornava os resultados insatisfatórios, mais uma vez na história da cirurgia estrabológica. Foi então, que os cirurgiões tiveram que procurar novas opções e passaram a usá-las conforme suas preferências pessoais ou baseados em teorias empíricas preconizadas por seus criadores. Basicamente, passou-se a utilizar-se dois tipos de condutas:

a. Tenotomia dos retos mediais de ambos os olhos: essa conduta foi muito usada por *Panas, que a defendia dizendo que, sendo o estrabismo uma afecção bilateral, a correção cirúrgica deveria, também, ser bilateral. O mesmo dizia **Landolt, mas para argumentar a favor do avançamento em ambos os olhos ${ }^{(12,14)}$.

b. Tenotomia combinada com avançamento, com ou sem ressecção, no mesmo olho: essa conduta também logo angariou muitos adeptos, pois além da vantagem óbvia de operar-se somente um olho, tinha a vantagem de enfraquecer o músculo mais forte (reto medial) e fortalecer o músculo mais fraco (reto lateral).

Realmente, estava plantada, definitivamente, na estrabologia mundial, a polêmica cirurgia monocular versus cirurgia binocular, ambas as condutas com seus seguidores, que, infelizmente, optavam por uma delas por preferência pessoal e não por trabalhos científicos que demonstrassem a superioridade de uma técnica em relação à outra.

Com a tenotomia graduada, mormente a retroinserção com fixação escleral, a cirurgia estrabológica apresentou um grande avanço, ganhando mais previsibilidade e segurança ${ }^{(19)}$. No início o autor fez somente cirurgias monoculares, com recuo máximo de $5 \mathrm{~mm}$ do reto medial, mas, como as hipocorreções foram aparecendo nos grandes ângulos, ele passou a operar os retos mediais de ambos os olhos, nesses casos. Ele poderia, talvez, ter feito a cirurgia monocular com o recuo do reto medial e avançamento do reto lateral, com ou sem ressecção. Entretanto, preferiu a cirurgia binocular com recuo de ambos os retos mediais.

Nos anos que se seguiram, a técnica de Jameson foi sendo aperfeiçoada, tornando-se, praticamente, igual a que se faz em nossos dias (com melhorias somente pelas novas tecnologias), mas o limite de $5 \mathrm{~mm}$ de recuo foi-se mantendo. A cirurgia de avançamento foi perdendo terreno para a cirurgia de ressecção muscular, de modo que, nas décadas seguintes, as opções para a correção cirúrgica das esotropias, ficaram resumidas ao duplo

\footnotetext{
*Panas. Pathogénie et traitement du strabisme fonctionnet dit concomitant. Arch. d'Ophthalmol. 1898 apud $^{(12)}$

**Landolt Remarques sur l'avancement musculaire. Ann d'Opht. 1904. apud ${ }^{(12)}$
}

recuo dos retos mediais, à dupla ressecção dos retos laterais (ambas, cirurgias binoculares) e à cirurgia monocular de recuo do reto medial combinado com ressecção do reto lateral, no mesmo olho, chamada, simplesmente, cirurgia de recuo-ressecção. Posteriormente, a ressecção dupla de retos laterais foi perdendo adeptos, de modo que a polêmica acabou circunscrita aos duplos recuos de retos mediais, de um lado e à cirurgia de recuo-ressecção, do outro.

Atualmente, continua o dogma do limite de $5 \mathrm{~mm}$ de recuo para o reto medial e, relativamente à ressecção do reto lateral, outro dogma foi aparecendo qual seja a ressecção máxima de $7 \mathrm{~mm}$ (ou $8 \mathrm{~mm}$, eventualmente), de modo que a cirurgia monocular tem sido feita somente para os ângulos chamados de moderados, geralmente em torno de 35 a $40^{\Delta}$. Entretanto, mesmo para esses ângulos moderados, existem autores que indicam somente a cirurgia binocular, atuando em dois músculos ${ }^{(34)}$. Para ângulos maiores, esses autores já recomendam operar três ou quatro músculos, evitando, sempre, a cirurgia monocular.

Pode-se concluir, portanto, que a cirurgia monocular só não é praticada para as esotropias de grande ângulo, porque os autores limitam os recuos do reto medial em $5 \mathrm{~mm}$ (às vezes $6 \mathrm{~mm}$ ) e as ressecções em $7 \mathrm{~mm}$ (às vezes $8 \mathrm{~mm}$ ) por receio de limitações nos movimentos oculares e perda de convergência. Entretanto, existem trabalhos na literatura com recuos e ressecções maiores, chegando alguns autores a 8, 9, ou mesmo $10 \mathrm{~mm}$ de retrocesso do reto medial(27-30,35-37), o mesmo acontecendo com as ressecções, chegando-se a $10 \mathrm{~mm}$ com frequência e às vezes até $12 \mathrm{~mm}^{(2,4-5,7,35-36)}$ e não foram encontradas grandes limitações de movimentos oculares, nem a perda da convergência.

O trabalho de Meirelles-Teixeira et al. ${ }^{(36)}$ refere-se à cirurgia monocular de recuo-ressecção para correção de esotropias de grande ângulo. Trata-se de um estudo retrospectivo onde foram revisados 309 prontuários, de janeiro de 1996 a fevereiro de 1999, dos quais foram selecionados 20 pacientes com ângulos iguais ou maiores que $60^{\Delta}$, amblíopes e com esotropia não acomodativa, operados com anestesia tópica e ajustes per-operatórios. Os recuos do reto medial chegaram a $9 \mathrm{~mm}$ e as ressecções a $10 \mathrm{~mm}$, sendo a maioria dos pacientes, adultos e três deles adolescentes. A cirurgia foi sempre no olho amblíope, preservando-se assim o olho de melhor visão.

Os resultados foram bons em 70,5\%, regulares em 17,6\% e ruins em $11,7 \%$ e as rotações binoculares foram zero e $-1,0$ na maioria dos casos, tendo somente dois casos com -2,0 do reto medial recuado, mostrando não haver as limitações esperadas, o que os autores atribuem à anestesia tópica e aos ajustes peroperatórios.

O trabalho de Corrêa, Bicas ${ }^{(35)}$, por outro lado, compara dois grupos de pacientes com esotropias entre 30 e $60^{4}$, submetidos à cirurgia monocular de recuo-ressecção (recuo máximo de $8 \mathrm{~mm}$ e ressecção máxima de $10 \mathrm{~mm}$ ), sendo que em um deles a anestesia usada foi a geral, não havendo reajustes operatórios e, no outro, a anestesia foi a tópica, sendo feitos reajustes pós-operatórios imediatos. Os resultados cirúrgicos foram semelhantes, no pós-operatório imediato, para ambos os grupos, mas, no terceiro e no sexto mês foram melhores, estatisticamente, para o grupo com anestesia geral.

\section{CONCLUSÃO}

Por consenso da grande maioria dos oftalmologistas, a correção cirúrgica das esotropias de grande ângulo é feita por meio de cirurgia binocular, atuando-se nos quatro músculos horizontais (ou pelo menos em três deles), devido à obediência a um parâmetro antigo da oftalmologia, que é o recuo 
máximo de $5 \mathrm{~mm}$ para o reto medial. Entretanto, pela revisão bibliográfica feita, vimos que muitos autores já praticaram valores acima dos $5 \mathrm{~mm}$ preconizados, tanto em cirurgias binoculares como em monoculares. Portanto, entendemos que a cirurgia monocular de recuo-ressecção, pode ser uma opção viável para correção de esotropias de grande ângulo, fazendose amplos recuos do reto medial e grandes ressecções do reto lateral, principalmente nos pacientes amblíopes, preservando-se, sempre, o olho de melhor visão.

\section{REFERÊNCIAS}

Kirby DB. Cirurgia do estrabismo. Rev Bras Oftalmol. 1948:6(2):135-44

2. Pueyo AC. Estrabismos y heteroforias. $2^{\mathfrak{a}}$ ed. Madrid: Paz Montalvo; 1958.

3. Ferreira LE. Cirurgia do estrabismo. J Bras Cir. 1962;1 (4):517-44.

4. Arruga H. Cirugía ocular. 5ª ed. Barcelona: Salvat; 1963.

5. Hugonnier R, Clayette-Hugonnier S. Strabismus, heterophoria, ocular motor paralysis: clinical ocular muscle imbalance. Saint Louis: C. Mosby; 1969.

6. Foschini RM, Bicas HE. Cirurgias de músculos retos horizontais: análise de planejamentos e resultados. Arq Bras Oftalmol. 2001;64(5); 523-34.

7. Moreira JB Cirurgia de estrabismo. Ars Curandi. 1974:5(Suppl):174-92

8. von Noorden GK. The history of strabismology. Belgium: J.P. Wayenborgh; 2002

9. Álvaro ME. Tratamento cirúrgico do estrabismo. Ophtalmos. 1940:9-24.

10. Gallo A. Histórico: a evolução do pensamento cirúrgico na correção do estrabis mo. In: Centro Brasileiro de Estrabismo. Memória do século XX: coletânea das publicações do Centro Brasileiro de Estrabismo. Belo Horizonte: RC; 2001, p.178-81.

11. von Noorden GK. Special lecture: history of strabismology. Presented at: $29^{\text {th }}$ European Strabismological Association; 2004 Jun 1-4. Meeting. Izmir p.24-5.

12. Mascarenhas BD. Tratamento cirúrgico do estrabismo funcional [tese]. Rio de Janeiro: Faculdade de Medicina do Rio de Janeiro; 1922.

13. Martins BJ. Dissertação sobre o estrabismo: seguida de algumas observações [tese]. Rio de Janeiro: Faculdade de Medicina do Rio de Janeiro: 1845.

14. Bulcão JL. Do valor do tratamento cirúrgico na cura do estrabismo [tese]. Porto Alegre: Faculdade de Medicina de Porto Alegre; 1927.

15. Munoz-Roiz JL. Especialidades quirúrgicas. In: Entralgo PL. História universal de la medicina. Barcelona: Salvat; 1974

16. Arruga H. Cirugía ocular. Barcelona: Salvat; 1946.

17. Duverger C, Velter E. Thérapeutique chirurgicale ophtalmologique. Paris: Masson et Cie; 1926.
18. Fuchs E. Trattato de oftalmiatria. Napoli: Casa Editrice Cav. Dott. V. Pasquale; 1895

19. Jameson PC. Correction of squint by muscle recession with scleral suturing. Arch Ophthalmol. 1922:20:166-81.

20. Malbran J. Estrabismos y paralisis: clínica y terapeutica. Buenos Aires: Oftalmológica Argentina; 1949.

21. Berens C, Alvaro ME. El ojo y sus enfermedades. São Paulo: Edigraf; 1956

22. Tanganelli SP. O estrabismo: sua história. In: Souza-Dias CR, Almeida HC. Estrabis mo. São Paulo: Rocca, 1998, p.12-3.

23. Helveston EM. Surgical management of strabismus. $5^{\text {th }}$ ed. Belgium: J.P. Wayenborgh; 2005

24. Blaskovics L, Kreiker A. Cirugía de los ojos. Buenos Aires: Salvat; 1947.

25. Parks MM. Ocular motility and strabismus. Virginia, USA: Harper \& Row; 1975

26. Hes JB, Calhoun JH. A new rationale for the management of large angle esotropia Pediatr Ophthalmol Strabismus. 1979;16(6):345-8.

27. Prieto-Diaz J. Large bilateral rectus recession in early esotropia with bilateral limitation of abduction. J Pediatr Ophtalmol Strabismus. 1980;17(2):101-5.

28. Prieto-Diaz J. Five year follow-up of large $(6-9 \mathrm{~mm})$ bimedial recession in the management of early onset, Infantile esotropia with Ciancia syndrome. Binocul Vis, 1986; (4):209.

29. Szmyd SM, Nelson LB, Calhoun JH, Spratt C. Large bimedial rectus recession in congenital esotropia. Br J Ophthalmol. 1985;69(4):271-4.

30. Nelson LB, Calhoun JH, Simon JW, Wilson T, Harley RD. Surgical management of large angle congenital esotropia. Br J Ophtalmol. 1987;71(5):380-3.

31. Damanakis AG, Arvandis PG, Ladar ID, Theodossiadis GP. $8 \mathrm{~mm}$ bimedial rectus recession in infantile esotropia of 80-90 prism diopters. Br J Ophthalmol. 1994; 78(11):842-4.

32. Vroman DT, Hutchinson AK, Saunders RA, Wilson ME. Two-muscle surgery fo congenital esotropia: rate of reoperation in patients with small versus large angles of deviation. J AAPOS. 2000:4(5):267-70

33. Good W, Hoyt CS. Strabismus management. Newton, MA: Butterworth-Heinemann; 1996.

34. Souza-Dias CR, Almeida HC. Estrabismo. Conselho Brasileiro de Oftalmologia. São Paulo: Rocca; 1998.

35. Corrêa BA Bicas HE Estudo comparativo de cirurgias monoculares para esotropias sob anestesia geral e sob anestesia tópica, com ou sem reajuste. Rev Bras Oftalmol. 1998;57(10):747-55

36. Meireles-Teixeira JA, Cunha RP, Mendonça TS. Resultado da correção cirúrgica de esotropias de grande ângulo, em portadores de baixa acuidade visual unilateral Arq Bras Oftalmol. 2000;63(5):365-8.

37. Rodriguez-Vásquez R. Retroinsercion amplia en cirugia de endotropias alternas Apresentado no: $4^{\circ}$ Congreso del Clade; 1974. México.

\section{ABO ELETRONNIBO}

\section{A versão eletrônica dos Arquivos Brasileiros de Oftalmologia com textos completos está disponivel em:}

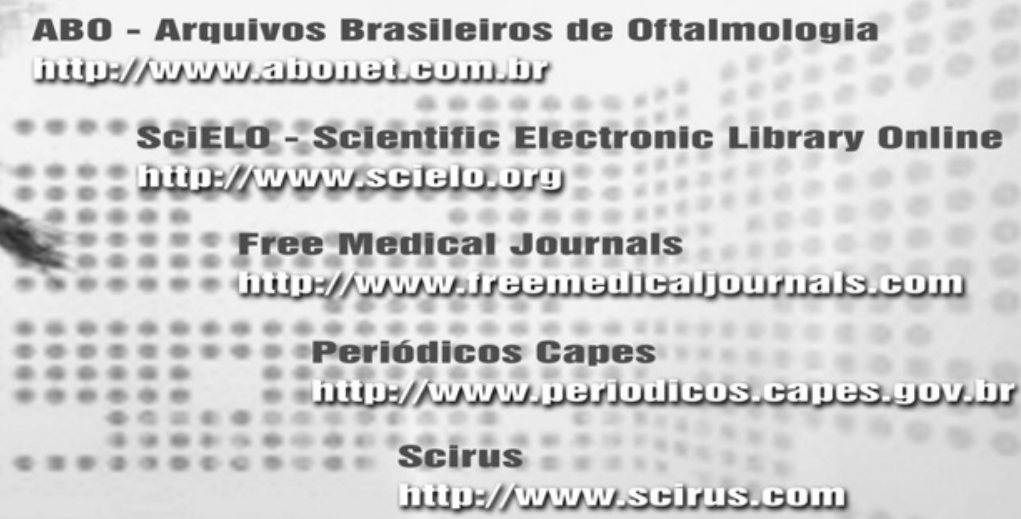

Free Medical Journals

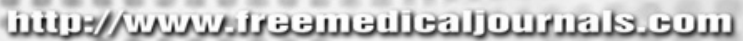

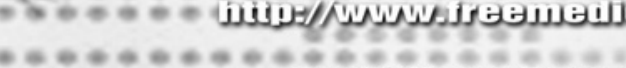

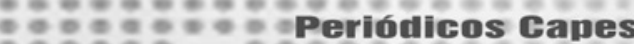

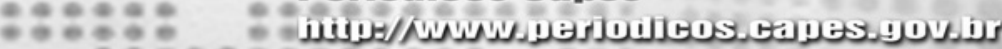

\&ats

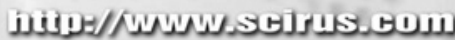

Copernifus

سن س 